\title{
A Mixed-Prediction based Method for Allocating Cloud Computing Resources
}

\author{
Qian Zhao ${ }^{1}$, Guangsheng Feng ${ }^{2}$, Rui Gao ${ }^{2}$ and Ke Han ${ }^{1}$ \\ ${ }^{1}$ School of Computer and Information Engineering, Harbin University of \\ Commerce, Harbin, China \\ ${ }^{2}$ College of Computer Science and Technology, Harbin Engineering University, \\ Harbin, China \\ \{zhaoqian, fengguangsheng, gaorui\}@hrbeu.edu.cn \\ hanke@hrbcu.edu.cn
}

\begin{abstract}
Due to lacking valid prediction for resource requirement, the existing application approaches for cloud computing resource could hardly achieve a high efficiency. According to this point, we propose a mixed-prediction based resource allocation approach in this paper, which is abbreviated as MPRA. This proposed MPRA employs FFT (Fast Fourier Transform) theory to determine the cyclical attribution. If there is no such attribution existed, the Markov chain is alternatively used to predict the tendency of resource requirement. The experimental results show that the proposed MPRA could predict the future resource requirement more precisely. Moreover, based on the prediction result, it could also allocate the virtual machine resource adaptively, decrease the number of occupied physical machines, and reduce the probability of violating the SLA (Service-level Agreement).
\end{abstract}

Keywords: Virtual machine, Resource allocation, Fast Fourier Transform, Markov Chain

\section{Introduction}

The traditional resource allocation and payment according to the configuration of the cloud computing platform cannot satisfy the increasing variety of end users and individual needs, and the payment mode according to the individual requirement has caused extensive concern [1]. The coarse-grained resource allocation is a typical method used by each cloud service provider [2, 3], such as Amazon, Google, etc. This method takes virtual machine as scheduling unit, and then increases or decreases virtual machines numbers to achieve the goal of load adaptive. However, this traditional way of resource allocation could cause the virtual machine scattered distribution, and physical machine resource occupation increasing and resource wasted easily [4-6]. As the booming of cloud users especially mobile users, this resource allocation methods not only hinder the improvement of resources utilization rate seriously, and cause the increase of operating costs rapidly [7-10].

Optimizing the placement of the virtual machine has become a hot research direction. The literature [11] abstracts cloud platform virtual machine placement problem as a packing problem, it first takes CPU and memory resources as two dimensions, and then uses the Choco heuristic algorithm to find the optimal solution of the virtual machine placement. However, the complexity of the algorithm becomes too large with the dimension increasing. To overcome this limitation, a multi-parameter heuristic algorithm is proposed in [12], and it considers CPU, memory, network bandwidth resources, and other factors with an acceptable time 
complexity. This method only increases cloud resources utilization efficiency, but does not take the task characteristics and user preferences into account. Given this limitation, a task oriented resource allocation method is proposed [13] by analyzing the task matrix of the user preferences and resource requirements. This method preallocates resources according to the demand list, and improves the precision of the allocation of resources with the assistance of Induction Deviation Matrix.

The typical algorithms are usually based on the dynamic need to adjust the virtual machine numbers, but the new virtual machine generally need ten minutes to allocate resources, which influences the user experience seriously. Aiming at this problem, a kind of online real-time data mining system is proposed [14] to predict virtual machine resource request, which serves as the basis of virtual machine preconfiguration. To reduce the violation of the Service Level Agreement (SLA), the works $[15,16]$ propose a resource dynamic adjustment based on prediction respectively, the former one realizes the prediction of virtual machine memory usage by auto regression, and the latter adjusts the virtual machine resources by periodic load change predictions, but the prediction accuracy is not high.

However, the existing researches still have some deficiencies, the existing resource prediction and allocation algorithms do not distinguish different categories of service resources demand, and prediction accuracy is not quite ideal. To solve these problems, this paper designs an adaptive allocation method, the Mixed Prediction based cloud platform Resource Allocation (MPRA), which is able to distinguish service resources demand characteristics and meanwhile judge periodic characteristics of service demand based on FFT theory. The proposed method could achieve the goal of improving system resource utilization and reducing the violation of SLA simultaneously.

\section{MPRA: Mixed Prediction based Cloud Platform Resource Allocation Model}

Let $v=\left\{v_{1}, \ldots, v_{m}\right\}$ denote the current $m$ virtual machines that cloud platform system has allocated, and $s_{i}$ denote the amount of resource allocated to virtual machine $v_{i}$. There are $l$ physical machines present in the cloud platform system, which is expressed by $p=\left\{p_{1}, \ldots, p_{l}\right\}$, and $R(v, p)$ represents the mapping relationship between the virtual machine set $v$ and the physics machine set $p$. Because any kind of services has its own resource requirements, the primary task of MPRA is first to record those changes of services demand. When a certain number of sample data is obtained, the MPRA employs FFT theory to determine the service period, and thus categorizes those services into two classes, named periodic service and aperiodic service. For resource requirements with periodic characteristics, its shortest cycle is obtained by FFT, while the resources requirements with aperiodic characteristics are predicted by Markov process.

Suppose $X=\{X(1), \ldots, X(L)\}$ as the $L$ services provided by the cloud platform, and the resources interval is $\left[X_{\min }^{(t)}, X_{\max }^{(t)}\right]$. Since the cloud platform lacks a priori knowledge and history of the initial access, MPRA will allocate the service resource with $X_{\max }^{(t)}$. After MPRA obtaining $w$ resource usage samples, i.e., $U^{(l)}=\left\{u_{1}^{(l)}, \cdots, u_{w}^{(l)}\right\}$, the resource demand forecasting process will be triggered: $U^{(l)}$ will be transformed by FFT, where time interval with significant amplitudes represents the cycle of request for the resource. However, the minimum one $f_{d}^{(l)}$ will be chosen as the main period frequency if there are several amplitudes greater than the average. Thus, it follows the maximum period of the resource request $1 / f_{d}^{(l)}$. For a main frequency $f_{d}^{(l)}$, the cycle window size of $U^{(1)}$ is:

$$
Z^{(l)}=\left(f_{d}^{(l)}\right)^{-1} \times r
$$


where $r$ is the sampling rate. If all types of service resource are with the same sampling rate, the time series will be dispersed into $Q=\left[w / Z^{(l)}\right]$ sub-time-series:

$$
\begin{aligned}
& \left\{\begin{array}{l}
U_{1}^{(l)}=\left\{u_{1}^{(l)}, \cdots, u_{Z^{(l)}}^{(l)}\right\} \\
\left\{U_{2}^{(l)}=\left\{u_{Z^{(l)}+1}^{(l)}, \cdots, u_{2 Z^{(l)}}^{(l)}\right\}\right. \\
\cdots \\
U_{Q}^{(l)}=\left\{u_{(Q-1) Z^{(l)}+1}^{(l)}, \cdots, u_{Q Z}^{(l)}\right.
\end{array}\right\}
\end{aligned}
$$

Based on the dispersed period window, a similarity test is conducted for any two windows. If the Pearson correlation coefficient of any two windows $U_{i}$ and $U_{j}$ closes to 1 and their average values are approximately equal, it is determined that the resource request of the service is in accordance with the cycle $Z(l)$. Then, the DWT (Dynamic Time Warping) technology is used to get the resources required sequence of the service $X(l)$ in the recent cycle, which makes the mapping distance between it and the current requiring resource sequence shortest. Thus, the resource demand of service $X(l)$ at time $t$ is $F^{l}(t)$.

However, when the service $X(l)$ does not have cyclical characteristic, we use Markov chain to predict the resource requirement at time $t$ in the paper. In order to use Markov theory, the resources required state of the service $X(l)$ is equally divided into $n=\left[\left(X_{\min }^{(l)}-X_{\max }^{(l)}\right) / I\right]$ intervals in advance, which is used to distinguish the state of the resource requirements in different conditions. Correspondingly, each resource demand interval represents a state of the resource or service status, and resource requirement for each state takes the mean value of the interval. Therefore, the changes of the resource requirement of the service can be considered as a time series $X(l)(t)(t=1,2, \cdots w)$. Resource requirement for each time point belongs to the different state of resource requirements. Then, we employ statistical technology to calculate the transition probability matrix of the Markov process.

$$
p^{(l)}=\left[p_{i j}^{(l)} \mid i=1, \cdots, n ; j=1, \cdots, n\right]
$$

where $p_{i j}^{(l)}=m_{i}^{(l)} / m_{i j}^{(l)}$ is the transition probability from state $s_{i}^{(l)}$ to state $s_{j}^{(l)}, m_{i}^{(l)}$ represents the numbers that the state $s_{j}^{(l)}$ appears at different time, and $m_{i j}^{(l)}$ represents the numbers state $s_{i}^{(l)}$ transfer to state $s_{j}^{(l)}$. So we can obtain the state transition probability matrix of this service $p^{(l)}$.

Based on this, MPRA predicts the short-term resource requirements by constructing a discrete finite-state Markov chain model. Provided that the Markov chain is homogeneous, any system status probability in time $t$ can be calculated by $\mathrm{C}-\mathrm{K}$ equation, and it follows the possible state prediction of the service:

$$
\pi_{t}^{(l)}=\pi_{t-1}^{(l)}\left(p^{(l)}\right)=\pi_{t-2}^{(l)}\left(p^{(l)}\right)^{2}=\cdots=\pi_{0}^{(l)}\left(p^{(l)}\right)^{t}
$$

where $\pi_{0}^{(l)}$ and $\pi_{t}^{(l)}$ is the probability distribution at the initial time and at time $t$ of $X(l)$ respectively. According to the current state $\pi_{0}^{(l)}$, the probability in state $s_{j}^{(l)}$ after the time $t$ can be inferred. Therefore, the forecast value of the resource requirements of the service at time $t$ is $\pi_{0}^{(l)}\left(p^{(l)}\right)^{t}$. In summary, the resource requirements of the service $X(l)$ at time $t$ can be forecasted as: 


$$
R^{(l)}(t)=\left\{\begin{array}{l}
F^{(l)}(t), \quad \text { if } X^{(l)} \text { has cyclical characteristics } \\
\pi_{0}^{(l)}\left(p^{(l)}\right)^{t}, \quad \text { if } X^{(l)} \text { has M arkov property }
\end{array}\right.
$$

\section{Resource Adaptive Allocation Algorithm}

The resource requirements trend of virtual machine can be obtained by the above demand forecasting. Suppose $S_{i}\left(\cup_{i=1}^{m} S_{i} \subseteq X\right)$ as the service set of the virtual machine $v_{i}$, and the resource demand forecasting of virtual machine $v_{i}$ at $t$ is as follows.

$$
R\left(t, v_{i}\right)=\sum_{X^{(t)} \in S_{i}} R^{(l)}(t)
$$

Assume that the resource allocation amount $S_{i}$ is allocated to the virtual machine $v_{i}$, the available resource amount in current physical machine is $R$, and the virtual machine resources usage expectation and variance are $\mathrm{E}\left(R\left(t, v_{i}\right)\right)$ and $\mathrm{D}\left(R\left(t, v_{i}\right)\right)$ respectively. Compensation value $U_{a d d}$, a linear function about the variance $\mathrm{D}\left(R\left(t, v_{i}\right)\right)$, is required to compensate according to the forecasting volatility. The larger the volatility is, the bigger the compensation value. According to the mapping relationship $R(v, p)$ between the physical machine and the virtual machine, all the virtual machines within the same physical machine will be arranged into an adjustment queue $Q_{1}$. The specific resource allocation algorithm RA is shown in Figure 1.

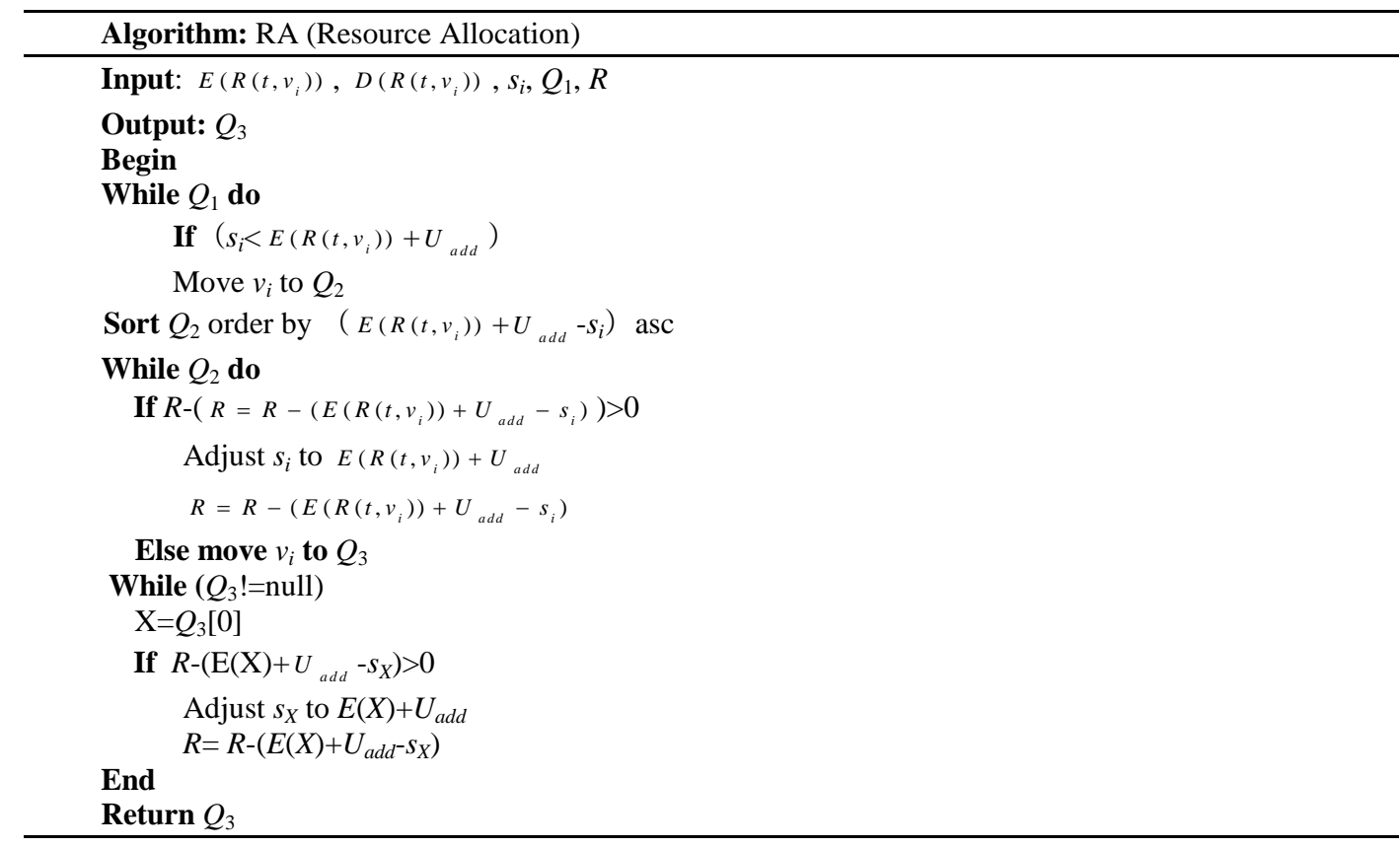

Figure 1. Resource Allocation Algorithm RA

In algorithm RA, traverse $Q_{1}$ first of all. If $S_{i}<\mathrm{E}\left(R\left(t, v_{i}\right)\right)+U_{\text {add }}, v_{i}$ will be put into the queue $Q_{2}$ with an ascending order. In this process, the total complexity of traverse $Q_{1}$ and sort $Q_{2}$ is $\mathrm{O}(n)$. Then, RA traverses $Q_{2}$. If the remaining resources $R$ of the physical machine can meet the demand of adjustment, $S_{i}$ will be adjusted to $\mathrm{E}\left(R\left(t, v_{i}\right)\right)+U_{\text {add }}$. Otherwise, the virtual machine will be put into the adjusting queue $Q_{2}$. Therefore, the complexity of traverse $Q_{2}$ is $\mathrm{O}(n)$. At last, poll the remaining 
resources $R$ of the physical machine. If $R$ can satisfy the resource requirements of the first element in $Q_{3}$, then adjust resources. Therefore the time complexity of RA is $\mathrm{O}(n)$.

If the current resource allocation amount exceeds the forecast, resource will be recycled timely and the recycling algorithm Resource Recycle (RR) is shown in Figure 2. In this procedure, RR first traverses $Q_{1}$. If it is found that the current allocated resources beyond the forecast, then virtual machine $v_{i}$ will be put into the queue $Q_{4}$, and sorted $Q_{4}$ according to the descending order. Secondly, poll $Q_{3}$ to judge whether it is null. There is no any processing if it is empty, otherwise the resource of the first element of $Q_{4}$ will be adjusted to $\mathrm{E}(s X)+U_{\text {add }}$. Thus, the time complexity of RR is $\mathrm{O}(n)$.

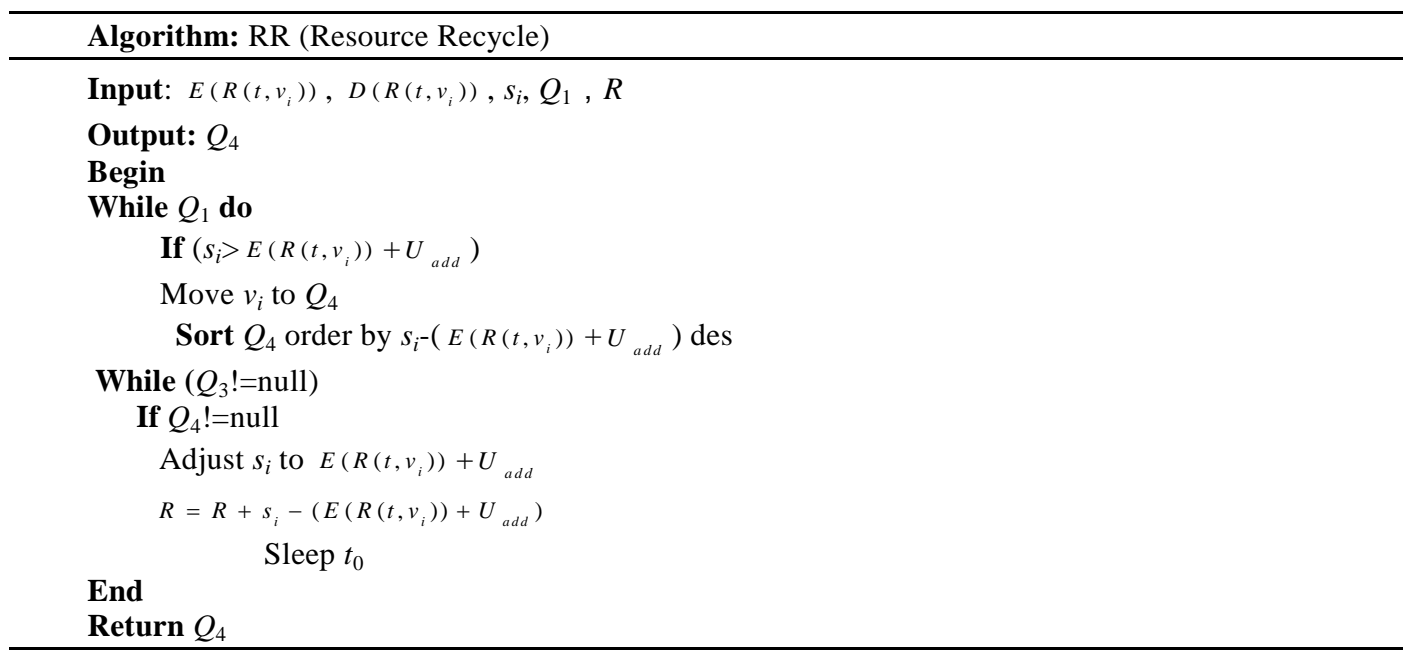

Figure 2. Resource Recovery Algorithm RR

\section{Experiment and Analysis}

\subsection{The Virtual Machine Resource Allocation Experiment}

The objective of this experiment is to test the validity of resource allocation method MPRA. We consider CPU and memory as the resource allocation objects, observe the virtual machine load changes over time, and also compare with ARMA [9] that is typical fixed resource allocation. The experiment used the Xen4.0 virtual machine platform, deployed in the 16 core CPU, 4GB memory's server, CentOS 64 bit version 5.2 as the virtual machine.

In the experiments, we first build two guest virtual machines VM1 and VM2 in the virtual machine platform, and each guest virtual machine is assigned a complete physical kernel and 512M memory to simulate physical machine with fixed resources. Then, VM1_sub and VM2_sub are built as the Web Servers. Moreover, aperiodicity application $\mathrm{AP}_{-} 1$ and periodic application $\mathrm{AP}_{-} 2$ are simulated by RUBis through importing ClusterData2011_10.

As shown in Figure 3, the curve in the fixed allocation algorithm reflects the actual change of requirement for service resources. In the fixed allocation algorithm, the CPU utilization rate is $40 \%$ in initial time, and then varies between $25 \%-50 \%$. Therefore, the physical CPU resource allocated to this virtual machine is sufficient. But in $8 \mathrm{~min}$, the ARMR algorithm triggers dynamic resource allocation, where the CPU utilization rate increases close to $80 \%$ with a deduction of physical CPU resource in $28 \mathrm{~min}$. It is obvious that the system may be corrupted anytime under such a dangerous state. The MPRA has a consistent tendency with fixed allocation until it collects a certain number of samples. The dynamic resource allocation 
mechanism is triggered in in $8 \mathrm{~min}$ and $16 \mathrm{~min}$, and CPU utilization of virtual machine remains about $70 \%$ after $22 \mathrm{~min}$. Thus, the fixed allocation leads to a great waste of physical CPU resources in contrast with MPRA.

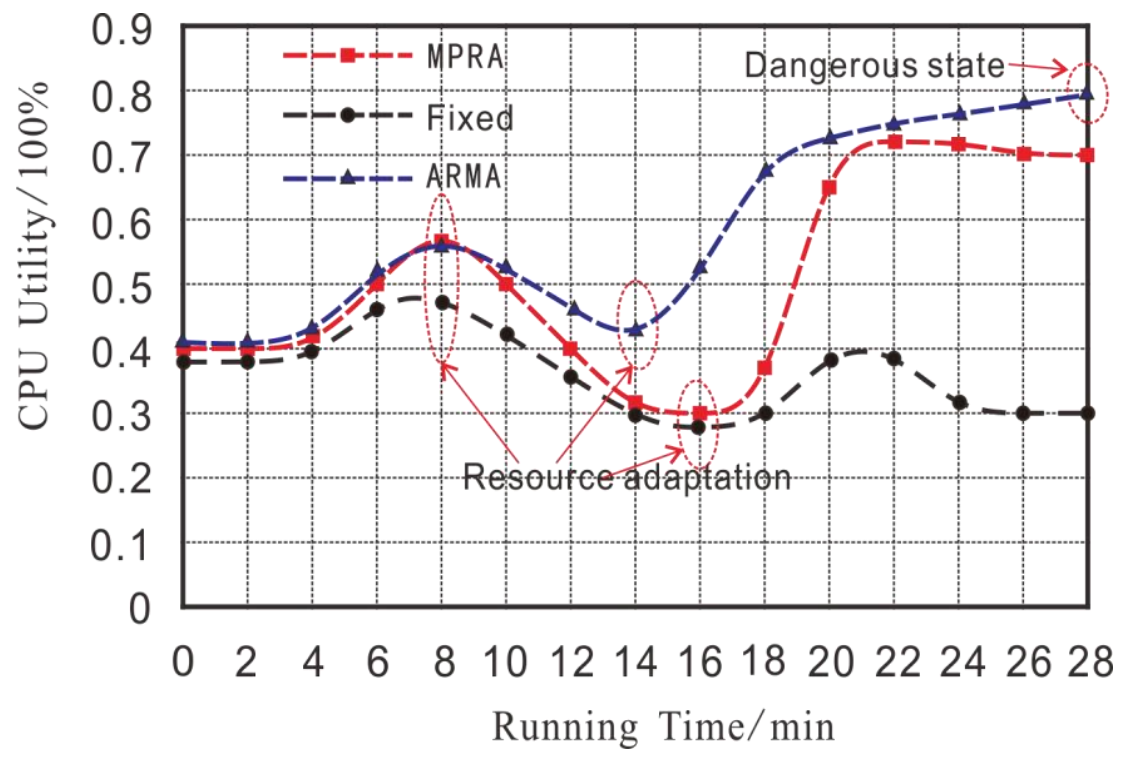

Figure 3. VM1 the Utility of CPU

Figure 4 shows the memory utilization rate of VM1. Similar to CPU resource, the fixed algorithm curve reflects the actual memory utilization which varies in the range of $40 \% \sim 55 \%$. Therefore, the memory resource is abundant with fixed allocation. ARMA algorithm adjusts memory resource in $8 \mathrm{~min}, 14 \mathrm{~min}$ and $26 \mathrm{~min}$. correspondingly, the memory utilization rate increases to $85 \%$ in $26 \mathrm{~min}$ because of inaccurate prediction, which may lead to a collapse event during the service process. The MPRA has accumulated enough prediction samples in $12 \mathrm{~min}$ so that the utilization rate of memory remains ideal state about $70 \%$.

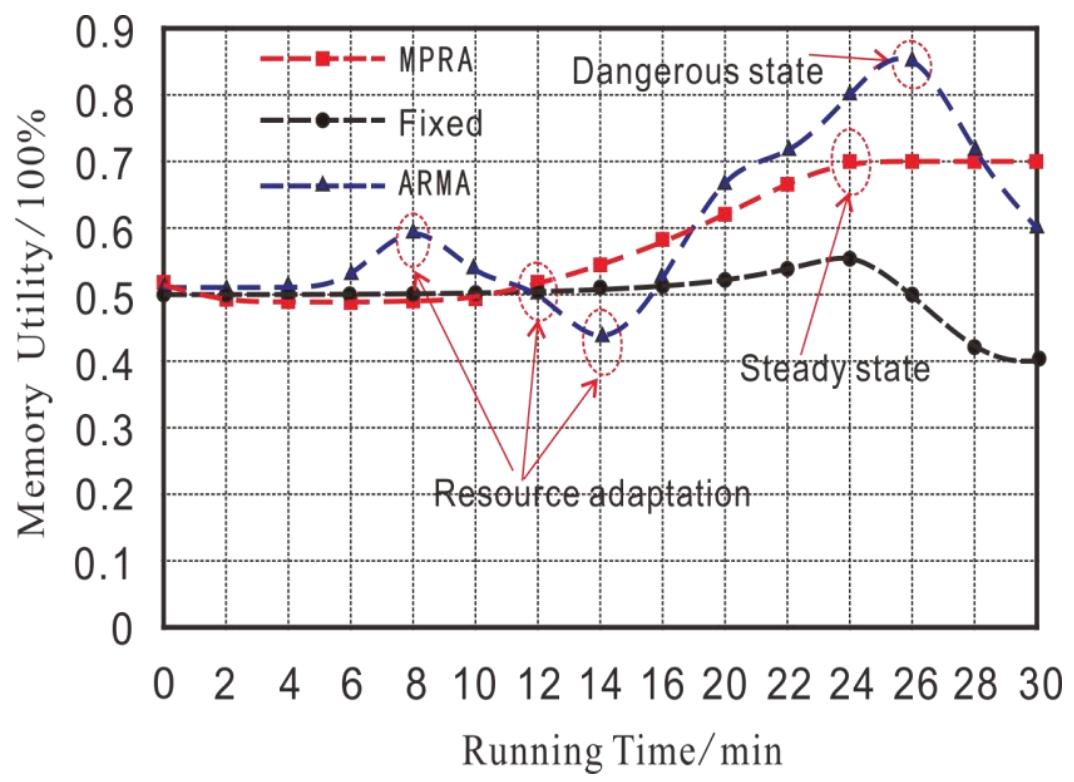

Figure 4. VM1: the Utility of Memory 
Figure 5 and Figure 6 are the CPU and memory utilization rates of VM2 where AP_2 serves as the requesting flow. In the fixed allocation, two kinds of resource allocation are abundant, and the corresponding utilization rates vary between 40\%-50\% and 45\%-55\% respectively. Because of the periodic characteristic of AP_2, ARMA and MPRA are able to get the ideal utilization rates in first $10 \mathrm{~min}$ similarly. After that point, the MPRA began to adjust the allocation of resources in $t_{1}$, and ARMA started later in $t_{2}$. Thus, the ARMA has a longer delay in reflecting the changes of service resource, which results lower resource allocation efficiency than MPRA.

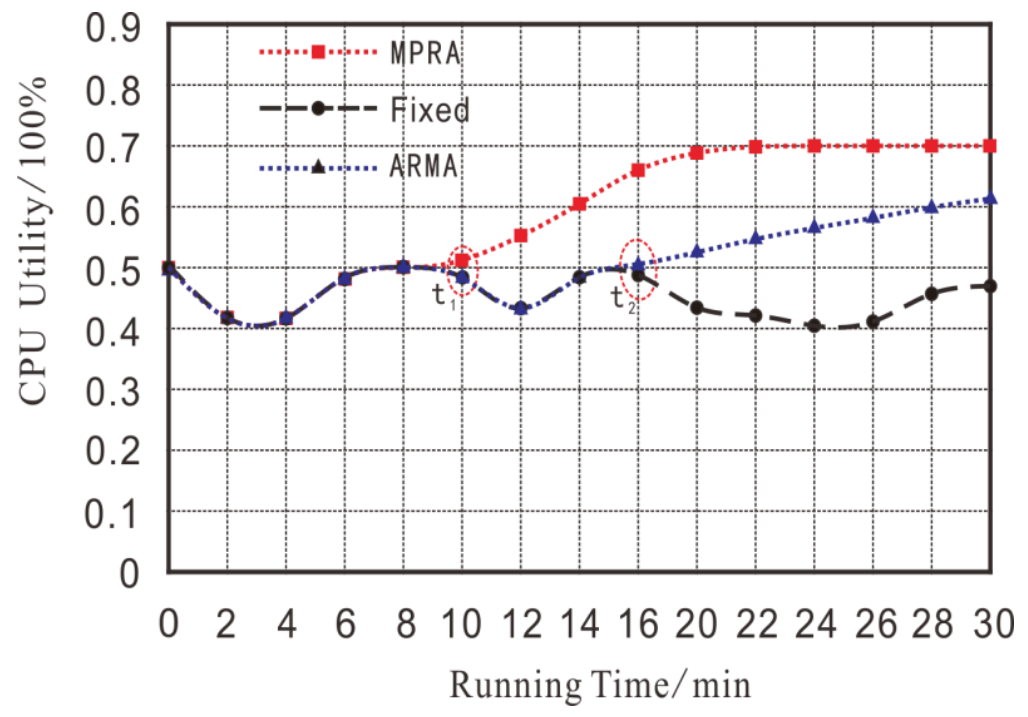

Figure 5. VM2: the Utility of CPU

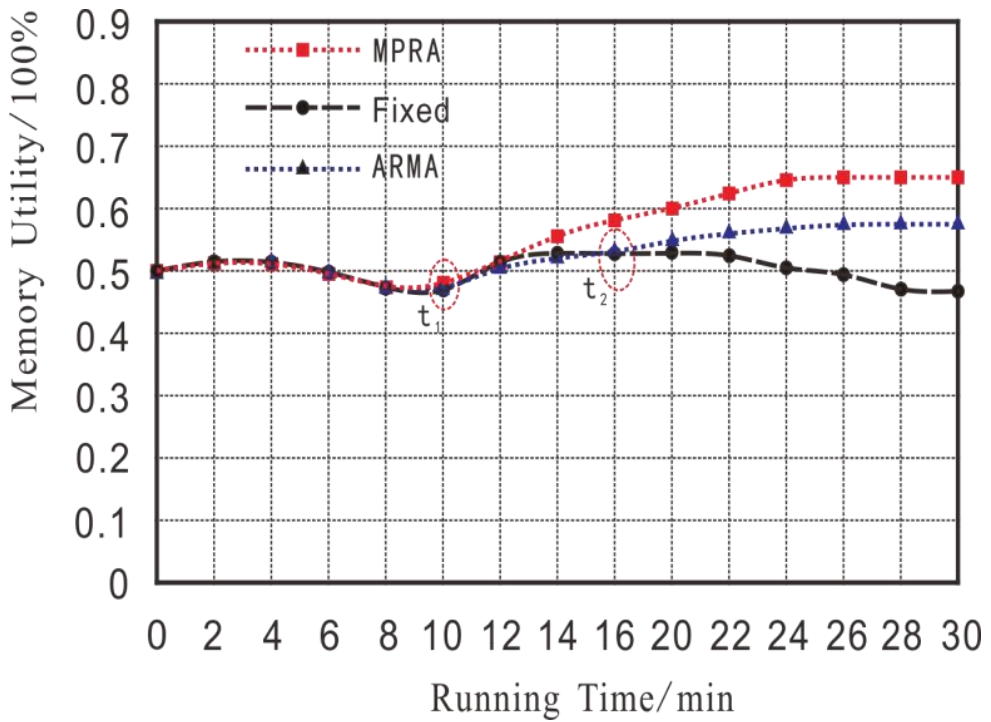

Figure 6. VM2: the Utility of Memory

In order to compare the prediction accuracy, the following experiments terminate the resource allocation process, and only collect the forecast data, in which the first $8 \mathrm{~min}$ data is considered as the prediction samples. The experiment results are shown in Figure 7 and Figure 8. Due to the system state influencing, the obtained data is not completely consistent with the former experiments. It is obvious that the MPRA has higher prediction accuracy than ARMA whether it is periodic or aperiodic service. When the service 
requests are periodic, both of two methods achieve a higher accuracy in contrast to aperiodic services.

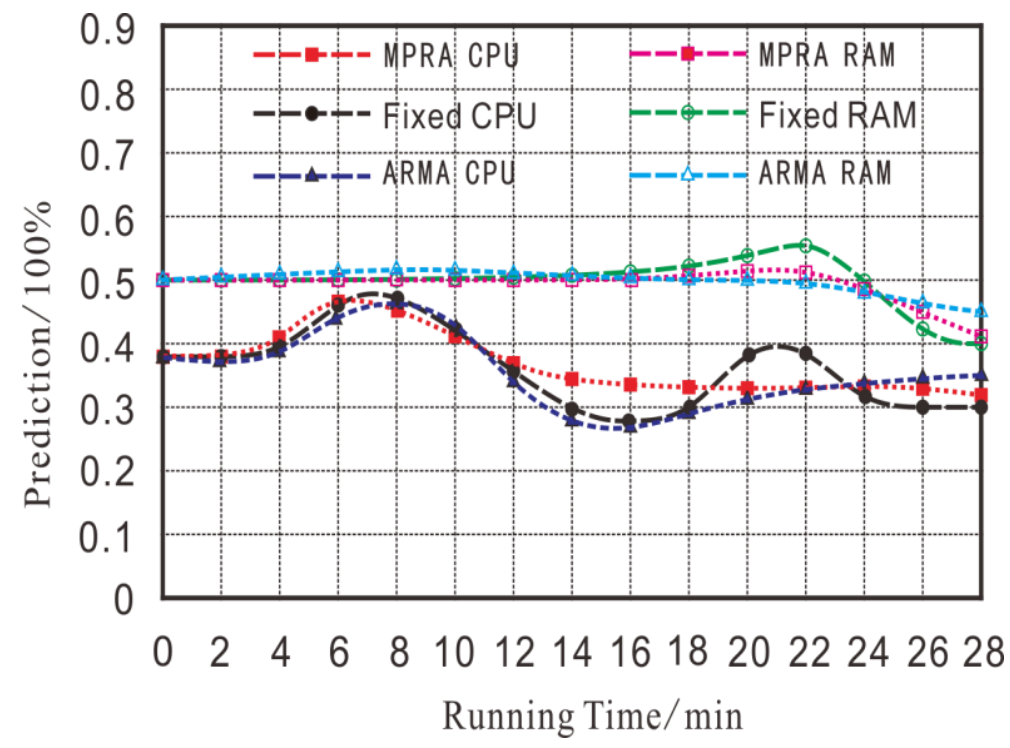

Figure 7. The Rate of Resource Predicted by the AP_1

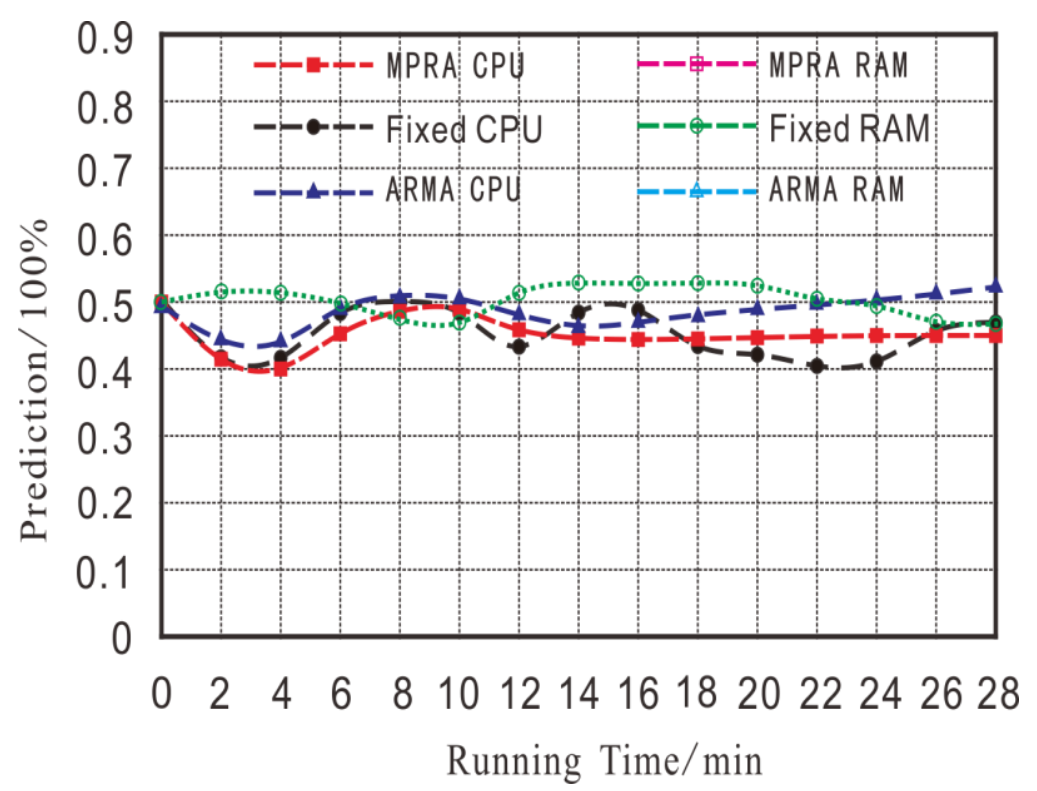

Figure 8. The Rate of Resource Predicted by the AP_2

\subsection{CloudSim based MPRA Simulation Analysis}

In order to test the occupancy of physical machine, average load of virtual machines and the number of violating SLA, the following experiments are deployed on CloudSim simulation platform with ClusterData2011_1. In the meanwhile, the load on 25 virtual machines in a month has been recorded. In this simulation, the CPU resource is taken as the main criterion, whose utilization between $0 \%-50 \%$ is considered as lower load, between $50 \%-80 \%$ as reasonable load, more than $80 \%$ as high-load, and more than 90 percent as a violation of the SLA threshold.

According to the results shown in Figure 9 and Figure 10, the load of virtual machine in FRA [10] is relatively balanced, where the number of low load is more than the others. On the contrary, the load of virtual machine in MPRA is mainly within a reasonable load 
range and only a little of low or high load exist. Therefore, the MPRA method can achieve higher resource utilization than FRA.

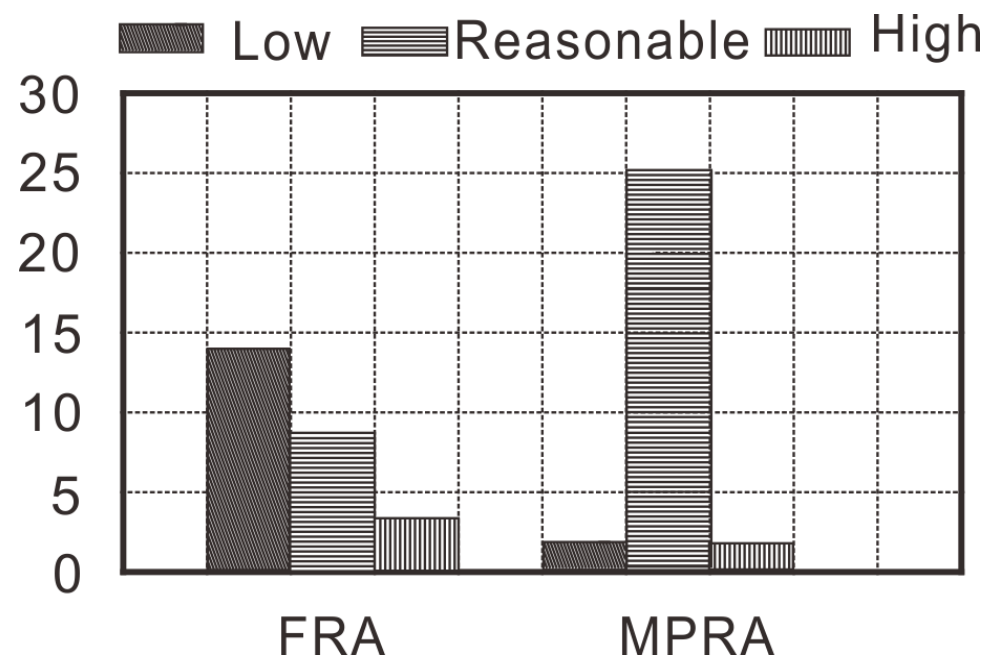

Figure 9. The Average Load of Virtual Machine

As shown in Figure 10, the virtual machines are assigned to the 11 physical machines in the initial stage. With the process of virtual machine migration, the number of physical machines is increasing correspondingly in FRA, which results in a high occupied ratio of physical machines and the consumption of physical machine resource is increasing. But in the beginning of MPRA, since the resource allocation is allocated according to a peak value, the number of the physical machine occupied by virtual machine almost remains a similar level. With the simulation going, this kind of physical occupancy is deducted. This is because that the MPRA could dynamically regulate virtual machine resources according to service request predication and each virtual machine could make full use of its allocated physical resources. Thus, the resource utilization of virtual machine remains at a high and reasonable level.

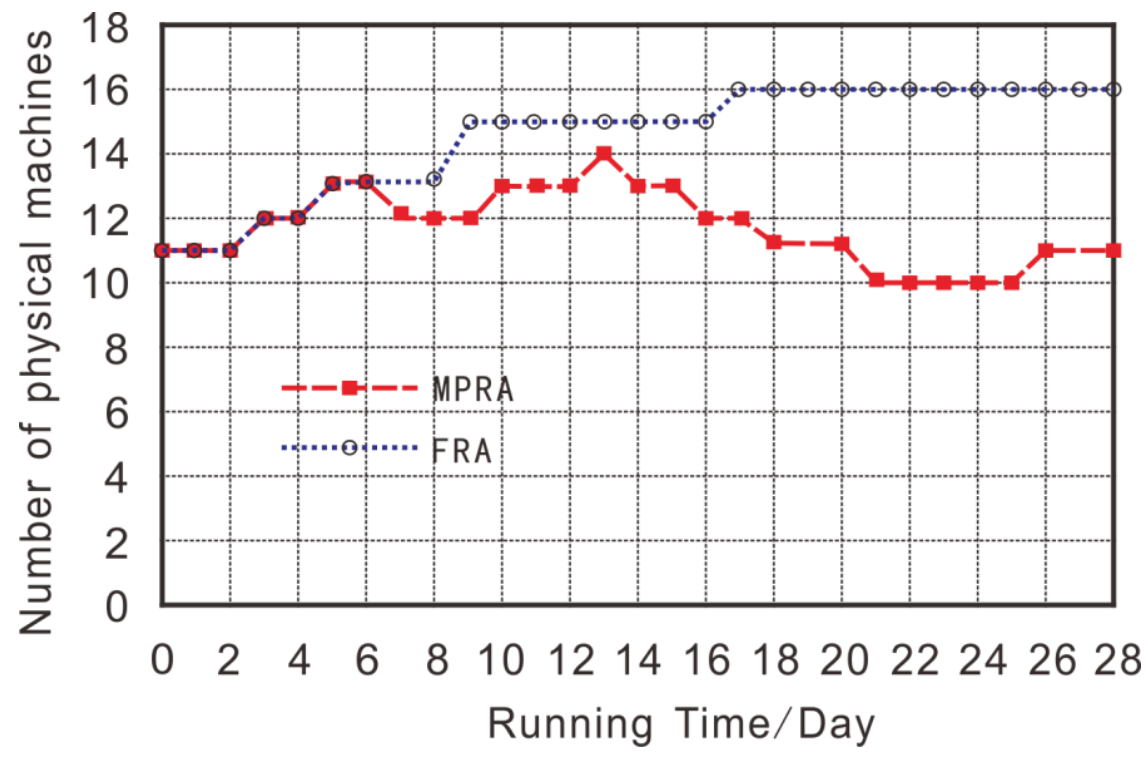

Figure 10. The Usage of Physical Machine

Table 1 shows the operating data within 27 days, the MPRA does not violate SLA in the whole operating time, while the FRA has occurred 12 times. 
Table 1. The Number of Violating SLA Contrast

\begin{tabular}{c|c|c|c|c}
\hline Violate SLA & $0-6$ & $7-13$ & $14-20$ & $21-27$ \\
\hline MPRA & 0 & 0 & 0 & 0 \\
\hline FRA & 4 & 3 & 3 & 2 \\
\hline
\end{tabular}

\section{Conclusion}

In this paper, the MPRA method is proposed to allocate cloud platform resource according to the periodic and aperiodic service requirement. This method first employs FFT theory to judge the periodic cycle and uses Markov process to predict aperiodic service resource requirement. This method obtains higher resource utilization efficiency than other traditional ones, and the number of violating SLA is deducted obviously. In the future, we will focus our research on the aperiodic service requirements.

\section{Acknowledgements}

This work is supported by the Research Fund for the Doctoral Program of Higher Education of China under Grant 20122304130002, the Natural Science Foundation of China under Grant 61370212 and 61402138, the Natural Science Foundation of Heilongjiang Province under Grant ZD 201102 and E201452, the Fundamental Research Fund for the Central Universities under Grant HEUCFZ1213 and HEUCF100601, the Support Program for Young Academic Key Teacher of Higher Education of Heilongjiang Province under Grant 1254G030, the Science and Technology Research Projects of Department of Education in Heilongjiang Province under Grant 12521135, and Postdoctoral Science Foundation of Heilongjiang Province under Grant LBH-210204.

\section{References}

[1] M. Armbrust, A. Fox and R. Griffith, "A view of cloud computing", Commun. ACM, vol. 53, no. 4, (2010), pp. 50-58.

[2] R. Buyya, C. S. Yeo and S. Venugopal, "Market-oriented cloud computing: Vision, hype, and reality for delivering it services as computing utilities", Proceedings of High Performance Computing and Communications, 2008. HPCC'08. 10th IEEE International Conference on, (2008), pp. 5-13.

[3] R. Buyya, C. Yeo and S. Venugopal, "Cloud computing and emerging IT platforms: Vision, hype, and reality for delivering computing as the 5th utility. Future Generation Computer Systems, vol. 25, no. 6, (2009), pp. 599-616.

[4] B. Grobauer, T. Walloschek and E. Stocker, "Understanding Cloud Computing Vulnerabilities", Security \& Privacy, IEEE, vol. 9, no. 2, (2011), pp. 50-57.

[5] Q. Duan, Y. Yan and A. Vasilakos V, "A Survey on Service-Oriented Network Virtualization Toward Convergence of Networking and Cloud Computing", Network and Service Management, IEEE Transactions on, vol. 9, no. 4, (2012), pp. 373-392.

[6] M. Sadiku, S. Musa and O. Momoh, "Cloud Computing: Opportunities and Challenges", Potentials, IEEE, vol. 33, no. 1, (2014), pp. 34-36.

[7] J. Lin, C. Chen and J. Chang, "QoS-Aware Data Replication for Data-Intensive Applications in Cloud Computing Systems”, Cloud Computing, IEEE Transactions on, vol. 1, no. 1, (2013), pp. 101-115.

[8] M. Kwang, "Agent-Based Cloud Computing", Services Computing, IEEE Transactions on, vol. 5, no. 4, (2012), pp. 564-577.

[9] N. Roy, A. Dubey and A. Gokhale, "Efficient Autoscaling in the Cloud Using Predictive Models for Workload Forecasting", Proceedings of Cloud Computing (CLOUD), 2011 IEEE International Conference on, (2011), pp. 500-507.

[10] S. Parikh, "A survey on cloud computing resource allocation techniques", Proceedings of Engineering (NUiCONE), 2013 Nirma University International Conference on, (2013), pp. 1- 5.

[11] H. Fabien, L. Xavier and J. Menaud, "Entropy: a consolidation manager for clusters", in Proceedings of the 2009 ACM SIGPLAN/SIGOPS international conference on Virtual execution environments. 2009, ACM: Washington, DC, USA. pp. 41-50. 
[12] V. Emeakaroha, I. Brandic and M. Maurer, "SLA-Aware Application Deployment and Resource Allocation in Clouds", Proceedings of Computer Software and Applications Conference Workshops (COMPSACW), 2011 IEEE 35th Annual, (2011), pp. 298-303.

[13] E. Daji, K. Gang and P. Yi, "The analytic hierarchy process: task scheduling and resource allocation in cloud computing environment", The Journal of Supercomputing, vol. 64, no. 3, (2013), pp. 835-848.

[14] A. Khan, X. Yan and T. Shu, "Workload characterization and prediction in the cloud: A multiple time series approach", Proceedings of Network Operations and Management Symposium (NOMS), 2012 IEEE, (2012), pp. 1287-1294.

[15] W. Zhao, Z. Wang and Y. Luo, "Dynamic memory balancing for virtual machines", SIGOPS Oper. Syst. Rev., vol. 43, no. 3, (2009), pp. 37-47.

[16] D. Menasce and M. N. Bennani, "Autonomic Virtualized Environments", Proceedings of Autonomic and Autonomous Systems, 2006. ICAS '06. 2006 International Conference on, (2006), pp. 28-28.

\section{Authors}

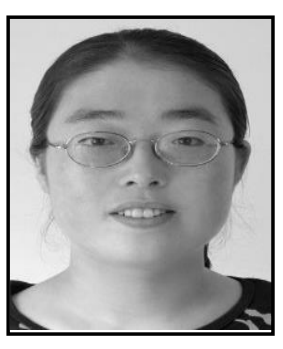

Qian Zhao, received the B.S., M.S. and Ph.D. degrees in computer science from College of Computer Science and Technology, Harbin Engineering University (HEU), China, in 2003, 2006 and 2010 respectively. Currently she is a LECTURER in School of Computer and Information Engineering, Harbin University of Commerce. Her current research interests include Ad Hoc network and wireless dependability.

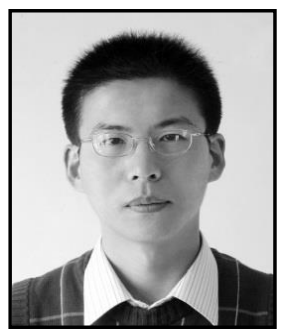

Guangsheng Feng, received B.S., M.S and D.S. degree from Harbin Engineering University in 2003, Harbin Institute of Technology in 2005 and Harbin Engineering University in 2009 respectively. Now, he is engaging in teaching and researching at Cognitive Networks. He is a full stuff at Harbin Engineering University. His research interests involve cross-layer design, information sensing and wireless channel access control.

Rui Gao, receives B.S. and M.S degree from Harbin Engineering University in 2012 and 2014. His research interests involve wireless dependability and wireless channel access control. 
International Journal of Grid Distribution Computing

Vol.8, No.2 (2015) 